\title{
BEAM DYNAMICS IN A STORAGE RING FOR NEUTRAL (POLAR) MOLECULES*
}

\author{
Glen R. Lambertson, University of California, Lawrence Berkeley National Laboratory, MS 71-159, \\ Berkeley, CA 94720 USA
}

\begin{abstract}
The force from a non-uniform electric field on the electric dipole moment of a molecule may be used to circulate and focus molecules in a storage ring. The nature of the forces from multipole electrodes for bending and focusing are described for strong-field-seeking and for weak-field-seeking molecules. Fringe-field forces are analyzed. Examples of storage ring designs are presented; these include long straight sections and provide bunching and acceleration.
\end{abstract}

\section{INTRODUCTION}

Molecules that have an electric dipole moment can be deflected, focused and accelerated by gradients of an electric field. We are studying the design of a storage ring for molecules that will have bunching and straight sections for experimental purposes. It will make available molecules in free space for scattering and spectroscopic measurements. In a circular ring constructed by Crompvoets et. al. [1], single bunches of molecules have been injected and observed to circulate for six turns. To provide a large current of molecules for injection at low velocity, these must be decelerated from a high-intensity source.

\section{FORCES}

The force on a molecule from a gradient in electric field magnitude is given by

$$
\vec{F}=-\vec{\nabla} W(E)=-\frac{d W}{d E} \vec{\nabla} E=P \vec{\nabla} E
$$

where $W$ is the potential of the polarized molecule in the field of magnitude $E$ and $P$ is its effective dipole moment in the field $E$. In contrast with a charged particle, here the force is a function of the magnitude of $E$ without regard for the direction of $E$. The function $W$ depends on the type of molecule and its rotational state and $P$ may be either in the direction of the field, positive, or opposed, negative. This polarity of $P$ makes the molecule either strong-field-seeking or weak-field-seeking. Examples of $W$ are shown in Fig. 1.

*Work supported by the Office of Science, U.S. Department of Energy under Contract No. DE-AC03-76SF00098

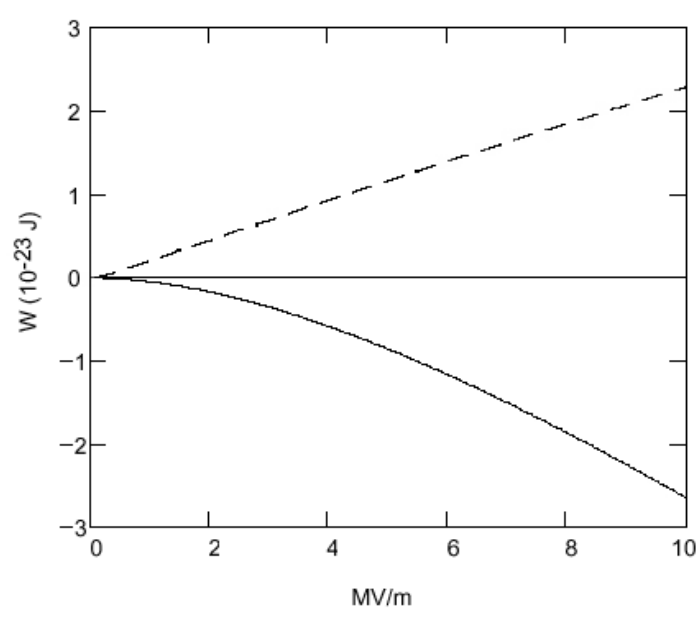

Figure 1: Potentials of methyl fluoride $(\mathrm{CH} 3 \mathrm{~F})$ in the ground state (solid curve) and of deuterated ammonia (14N2H3) in the $|\mathrm{J}, \mathrm{K}, \mathrm{M}\rangle=\mid 1,1,-1>$ state (dashed curve).

In a 2-dimensional field that is the gradient of the potential

$$
\Psi=E o\left[y+a_{2} x y+a_{3}\left(x^{2}-\frac{y^{2}}{3}\right) y\right]
$$

the forces on the $x$ and $y$ axes are

$$
\begin{aligned}
& F_{x}(x, 0)=P E o\left(a_{2}+a_{3} x\right) \\
& F_{y}(0, y)=P E o \frac{\left[\left(a_{2}\right)^{2}-2 a_{3}\left(1-a_{3} y^{2}\right)\right] y}{\sqrt{\left(a_{2} y\right)^{2}+\left(1-a_{3} y^{2}\right)^{2}}}
\end{aligned}
$$

The strength $E o$ of the dipole component determines the polarization $P$. In the absence of a quadrupole term, i.e. $a_{2}=0$, this biased sextupole provides focusing and defocusing much like a quadrupole does for a charged particle. A biased quadrupole, $a_{2}$ only, will provide an $x$ deflecting force and a $y$-defocusing force for strong-fieldseekers or $y$-focusing for weak-seekers. And with all three multipole components in the field, one may have focusing in both directions for weak-seekers but not this double-focusing for strong-seekers. While the forces for 
small excursions can be nearly linear on the axes, elsewhere they are not. This is shown in Fig. 2 where offaxis forces for $\mathrm{CH}_{3} \mathrm{~F}$ in a typical biased sextupole field are plotted. The addition of decapole and higher multipoles can alter the character of the nonlinearities. The advantages of this have not been fully explored.
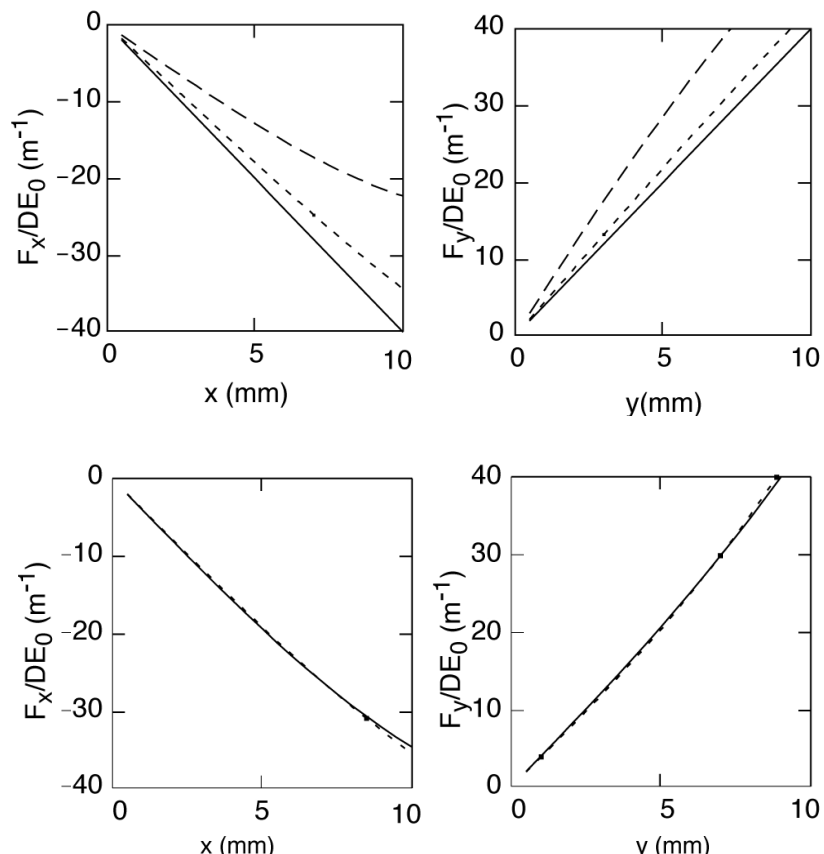

Figure 2: Force functions in a combined dipole and sextupole field. $F_{\mathrm{x}}$ at $\mathrm{y}=0,5$, and $10 \mathrm{~mm} . \mathrm{F}_{\mathrm{y}}$ at $\mathrm{x}=0,5$, and $10 \mathrm{~mm}$. (upper) $a_{3}=2000$. (lower) As in upper but with added decapole, $a_{5}=1350000$; off-axis forces superpose those on axis but all are now nonlinear.

The fringing fields at entrance and exit of a field electrode have a focusing effect in the direction of the $E$ field. This is in the defocusing sense for strong-fieldseekers and focusing for weak-field-seekers. If a molecule is moving in the $z$ direction at distance $y$ from the midplane in a known fringe field, one can numerically integrate $m \frac{d^{2} y}{d t^{2}}=\frac{-d W}{d E}$ to find the molecule's motion. A useful approximation for small $y$ requiring only the field on the midplane uses

$$
\frac{\partial E_{y}}{\partial y} \approx y \frac{\partial^{2} E_{y}}{\partial y^{2}}=y\left[\frac{1}{E_{y}}\left(\frac{\partial E_{y}}{\partial z}\right)^{2}-\frac{\partial^{2} E_{y}}{\partial z^{2}}\right]
$$

The overall focusing from passage through a length of changing field can then be expressed as a lens of focal length $f$ calculated as

$$
\frac{1}{f}=\int \frac{-1}{y} \frac{d^{2} y}{d z^{2}}=\int \frac{1}{m v^{2}} \frac{d W}{d E}\left[\frac{1}{E_{y}}\left(\frac{\partial E_{y}}{\partial z}\right)^{2}-\frac{\partial^{2} E_{y}}{\partial z^{2}}\right]
$$

Here $W, E_{y}$, and the velocity $v$ all vary with $z$ but this is still the most compact nearly-analytical way to obtain the effect of a fringing field. We see that by reducing $\partial E / \partial z$ by making the fringing length longer one could reduce an undesired fringe-effect, but that often conflicts with the desire to reduce the overall length of a molecule guide field.

To accelerate the molecule, a gradient, or $\Delta E$, in the direction of motion gives an energy change

$$
\Delta \frac{1}{2} m v^{2}=\Delta W(E)
$$

However, if a molecule is in a uniform field that changes with time, no acceleration results. Therefore if a molecule enters a field region and then the field is reduced, a strong-field-seeker will have gained velocity, or a weak-field-seeker will lose velocity. This process can be used repeatedly to make a large change in velocity or may be used with a ramped field pulse to act as a buncher.

\section{RING FOR A WEAK-FIELD-SEEKER}

As noted above, a bending field may be made to provide focusing simultaneously in the two transverse directions for a weak-field-seeking molecule. And the fringe fields are focusing. These actions aid greatly in designing a storage ring. But because the weak-seeker is not in the ground state, it is susceptible to transition to another state if in a weak and direction-changing field. Therefore the ring must have on the entire orbit a polarizing field of unchanging sign, but this is not difficult to provide.

The molecule in this example[2] is deuterated ammonia $\left({ }^{14} \mathrm{~N}^{2} \mathrm{H}_{3}\right)$ in the state $|\mathrm{J}, \mathrm{K}, \mathrm{M}\rangle=|1,1,-1\rangle$. The velocity in field-free space is $90 \mathrm{~m} / \mathrm{s}$, about 10 kelvin energy. The ring is in racetrack configuration as seen in Fig. 3. Arc radius is $20 \mathrm{~cm}$ and ring circumference is 3.36 meter. Straight sections are each 1 meter with $32 \mathrm{~cm}$ free for injection and access to the beam of molecules. Taking advantage of the double-focusing, the $90^{\circ}$ arc electrodes are continuous sextupoles in which the orbit is offset from zero field to provide the bending force; the gap is $12 \mathrm{~mm}$. An alternating-gradient triplet of biased sextupole lenses matches the beam motion from the arc into the straight section. The horizontal betatron tune is 5.25 and vertical, 5.20. the betatron functions and the dispersion are shown in Fig. 4. The field strengths and multipole coefficients of the guide-field elements are given in Table I. Transverse acceptances of the ring are $35 \mathrm{~mm}$-mrad horizontal and 71 mm-mrad vertical with $\pm 1 \%$ momentum spread. At the bunchers, the dispersion should be zero to avoid synchrobetatron coupling; to facilitate this the betatron phase advance is $2 \pi$ in each quadrant of arc. 


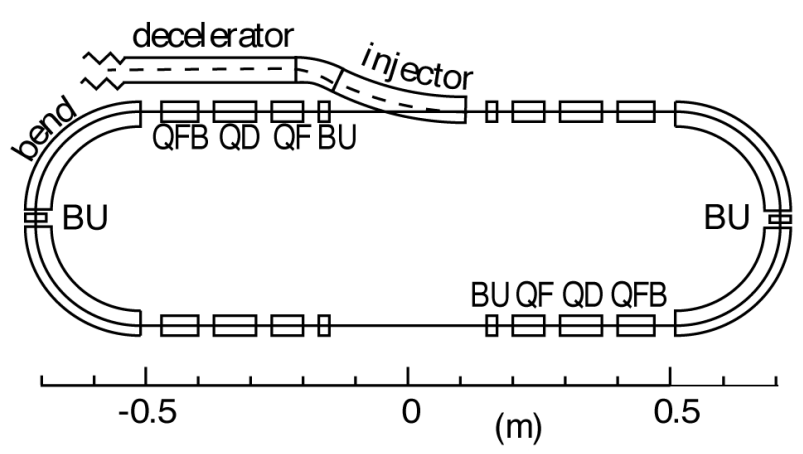

Figure 3: Layout of ring for deuterated ammonia at 90 $\mathrm{m} / \mathrm{s}$. Focusing lenses are labeled $\mathrm{Q}$, and bunchers, BU.

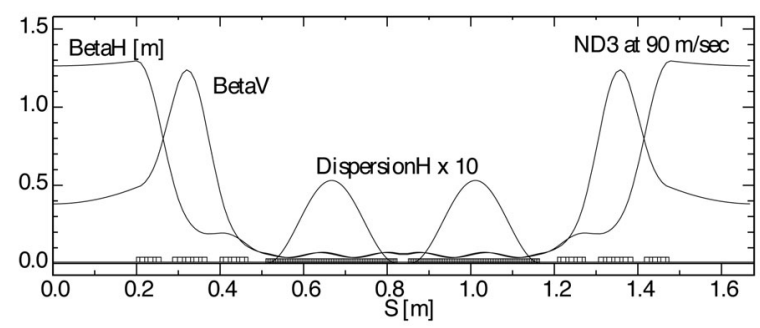

Figure 4: Betatron functions and dispersion of the ${ }^{14} \mathrm{~N}^{2} \mathrm{H}_{3}$ ring.

Table I: Field Strengths Eo, Multipole Coefficients, and Lengths $L$ of the Guide Fields in the ${ }^{14} \mathrm{~N}^{2} \mathrm{H}_{3}$ Ring

$\begin{array}{lccrr} & \begin{array}{c}E o \\ {[\mathrm{MV} / \mathrm{m}]}\end{array} & \begin{array}{l}a_{2} \\ {\left[\mathrm{~m}^{-1}\right]}\end{array} & \begin{array}{c}a_{3} \\ {\left[\mathrm{~m}^{-2}\right]}\end{array} & \begin{array}{c}L \\ {[\mathrm{~cm}]}\end{array} \\ \text { Bend } & 3.37 & 157 & 5667 & 31.4 \\ \text { QF } & 2.88 & 0 & -2000 & 6.0 \\ \text { QD } & 3.55 & 0 & -2000 & 8.0 \\ \text { QBF } & 4.30 & 0 & -2000 & 3.0\end{array}$

Injection is from a decelerator that brings molecules from $310 \mathrm{~m} / \mathrm{s}$ to $90 \mathrm{~m} / \mathrm{s}$ in a length of 3.4 meter. Bunches from the decelerator are injected through a bending electrode similar to that used in the arcs of the ring but pulsed off in a time that is much less than the $38 \mathrm{msec}$ circulation period to allow filling of a large fraction of the circumference.
The bunches are injected into 60 longitudinal buckets created by six parallel-plate bunching electrodes in the ring. The field in a buncher is pulsed at $1.6 \mathrm{KHz}$ with a triangular waveform that, as required, accelerates latearriving molecules and decelerates early ones. The field seen by an on-energy molecule is only $1 \mathrm{MV} / \mathrm{m}$ in a gap of $30 \mathrm{~mm}$. Bunches with $\pm 1 \%$ velocity spread that are captured in the ring are $7 \mathrm{~mm}$ long and spaced $56 \mathrm{~mm}$. Using a pulsed jet source into the decelerator, a single bunch contains $6.8 \times 10^{8}$ molecules and if all 60 buckets are filled, the circulating intensity would be $1.1 \times 10^{12}$ molecules per second. The bunchers may be used to decelerate the bunches by synchronously sweeping their frequency and the strengths of the ring guide fields. Without tune compensations, the velocity may be reduced to $60 \mathrm{~m} / \mathrm{s}$; with more controllable field parameters, this could be extended.

\section{RING FOR A STRONG-FIELD-SEEKER}

The molecule in this example[3] is methyl fluoride $\left(\mathrm{CH}^{3} \mathrm{~F}\right)$ in its ground state at $30 \mathrm{~m} / \mathrm{s}$. As seen in Fig. 1, the potential $W$ is quite nonlinear and in beam trajectory calculations this must be used to find the velocity and force at each point as the molecule moves through fields of various strengths. This is a greater factor at low velocity. For example, the $30 \mathrm{~m} / \mathrm{s}$ is the velocity in zero field; that rises to $33.2 \mathrm{~m} / \mathrm{s}$ in the $4 \mathrm{MV} / \mathrm{m}$ field of the bend electrodes.

This example ring has eight superperiods, each with arc and straight sections as shown in Fig. 5. The circumference is 9.85 meter and tunes are 13.37 horizontal and 10.40 vertical. Acceptances are $11 \mathrm{~mm}$ mrad horizontal and 21 vertical with $\pm 1.2 \%$ velocity spread. An arc has four cells with D/2-F-D/2 lattice; a cell has $\pi / 2$ phase advance in each plane. Each $F$ and $D$ sector in a cell has a dipole and a quadrupole component to provide the deflection and a sextupole term that reverses between $F$ and $D$. Field strength across the $8 \mathrm{~mm}$ aperture varies only about $1 \%$. The plate voltages of $\mathrm{F}$ and $\mathrm{D}$ are alike so that adjacent ends of successive sectors may be brought very close to reduce fringing fields in the transition region between them. A D-F-D triplet of lenses matches from arc to straight.

The force of gravity can displace by millimeters the vertical closed orbit if not compensated. A small vertical deflecting electrode in each triplet region reduces the displacement to about $0.1 \mathrm{~mm}$ at mid-straight as shown in Fig. 6. This correction may be adjusted when the velocity of the circulating molecules is changed. 


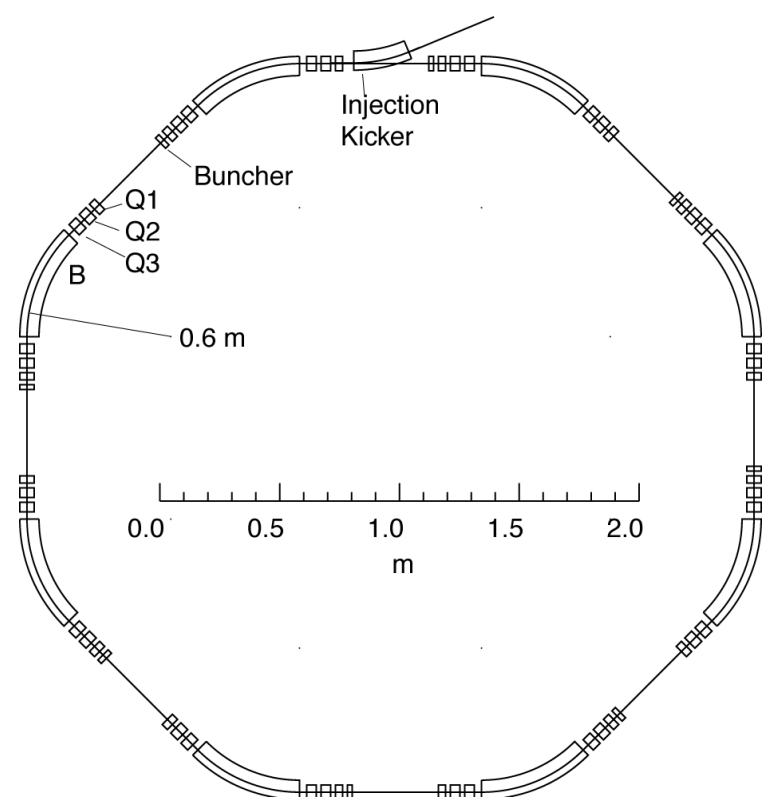

Figure 5: Layout of the storage ring for methyl fluoride.

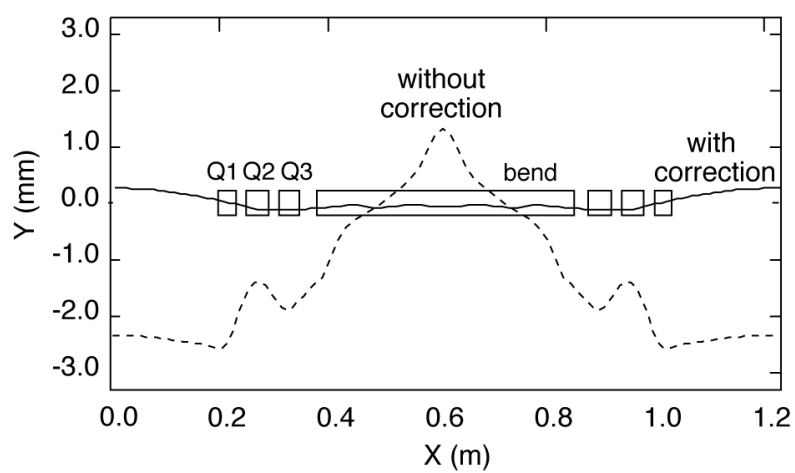

Figure 6: Displacement of the vertical closed orbit by gravity force, with and without compensation.

\section{DECELERATOR DESIGN}

A decelerator to provide low-velocity molecules for injection is a substantial part of a storage ring facility. It consists of a series of pulsed electrodes with uniform fields; in each of these, the field $E$ reduces the molecule's energy as in Eq. 6[3]. Many such electrodes are required; for methyl fluoride, 120 electrodes with pulsed field of 9 $\mathrm{MV} / \mathrm{m}$ are required to reduce $310 \mathrm{~m} / \mathrm{s}$ to $30 \mathrm{~m} / \mathrm{s}$. Between the pulsed electrodes one needs focusing lenses; in the case of strong-seeking $\mathrm{CH}_{3} \mathrm{~F}$, one lens for each pulsed decelerating electrode is required to confine the molecules transversely in the presence of the defocusing from fringe fields. Each electrode must have a length of uniform field for the bunch and this added to the lengths of fringing determines the bunch spacing and frequency at the exit end. A small slope on the top of the voltage pulse provides bunching action. If the same train of shaped pulses is applied to all electrodes, the bunch length varies as the square-root of velocity while bunch spacing is proportional to velocity. This allows the electrode lengths to be halved in an upstream portion of the array and greatly reduces its overall length. Some parameters of a 15.1-meter long decelerator for $\mathrm{CH}_{3} \mathrm{~F}$ are given in Table II. There are two electrodes per bunch spacing for velocity above $102 \mathrm{~m} / \mathrm{s}$.

Table II: Decelerator Parameters

$\begin{array}{lr}\text { Entering velocity [m/s] } & 310 \\ \text { Exit velocity [m/s] } & 30 \\ \text { Exit emittance [mm-mrad] } & 30 \\ \text { Exit bunch length [mm] } & 8 \\ \text { Electrode gap [mm] } & 6 \\ \text { Pulsed decelerating field [MV/m] } & 9 \\ \text { Lens dipole component [MV/m] } & 4.5 \\ \text { Entrance electrode length [mm] } & 124 \\ \text { Exit electrode length [mm] } & 24 \\ \text { Overall length [m] } & 15.1\end{array}$

\section{CONCLUSIONS}

For each manipulation with polar molecules there appears to be a method-different from that for charged particles and often with aggravating nonlinearities. But the low velocity brings the convenience of slow time scales for the components and small size. Electrodes for the guide fields are easy to fabricate and power. Storage ring concepts at this time are not highly developed. We need some experience as a preparation for more ambitious designs in response to the needs of users.

\section{ACKNOWLEDGEMENTS}

Designs and calculations reported here have been carried out in collaboration with Harvey Gould, Juris Kalnins, and Hiroshi Nishimura. Work on the synchrotron storage ring is supported by the Director, Office of Science, of the U.S. Department of Energy, and work on the linear decelerator is supported by the Director, Office of Science, Office of Basic Energy Sciences, of the U.S. Department of Energy, both under Contract No. DE-AC03-76SF00098.

\section{REFERENCES}

[1] F. Crompvoets, H. Bethlem, R. Jongma, and G. Meijer, "A prototype storage ring for neutral molecules", Nature 411, 174, (2001).

[2] H. Nishimura, G. Lambertson, J. Kalnins, and H. Gould, "Feasibility of a synchrotron storage ring for neutral polar molecules", to be published in RSI, LBNL-51597.

[3] H. Nishimura, G. Lambertson, J. Kalnins, and H. Gould, "Lattices for Milli-eV Neutral Molecules", in this proceedings, Portland, Oregon, May 2003. 